\title{
Compliance Assessment of Human Body Exposure to Wireless Power Systems
}

\author{
Cheikh Mohamed $^{1}$, Vigneau Guillaume ${ }^{2}$, Takacs Alexandru ${ }^{3}$ \\ ${ }^{1,2}$ Body and Security, Continental Automotive Group \\ 1 Av Paul Ourliac, Toulouse, France \\ Mohamed.cheikh@continental-corporation.com, guillaume.vigneau@continental-corporation.com \\ ${ }^{3}$ LAAS/Université de Toulouse \\ 7 Av Colonel Roche, Toulouse, France \\ takacs@laas.fr
}

\begin{abstract}
The interaction between wireless charging systems and the human body needs to be taken into account. Furthermore, a high power charging could lead to tissue heating and even irreversible tissue damage. In this paper, we propose an original approach to characterize the biological compatibility of the wireless charging system, from the transmitter coil to the human body: the influence of each element (Smartphone, coil orientation, human body, distance and charging power) on electromagnetic field is analyzed. The "specific absorption rate- SAR" estimations were carried out using a full environment model and a high resolution method. The electromagnetic level was measured and compared with simulation, showing a good agreement. The $10 \mathrm{~g}$ averaged SAR values are then compared with maximum permissible levels of the International Commission on Non-Ionizing Radiation Protection (ICNIRP) and the obtained results indicate that the inductive phone charging (from $100 \mathrm{kHz}$ to $200 \mathrm{kHz}$ ) are within the safety limits.
\end{abstract}

Keywords: FDTD, Wireless power systems, Qi, human safety, SAR, inductive transfer.

\section{Introduction}

Compatible Smartphone's can be charged wirelessly, saving space and without any tangled cables. Today, many leading Smartphone manufactures release their products equipped with built in wireless charging capability (e.g., Samsung, Nokia, LG, Nexus,...) [1]. Unit shipments of wireless charging receivers have tripled from 2014 to 2015, having reached over 160 million. Furthermore, consumer awareness of wireless charging technology has jumped from 34\% of all Smartphone owners in 2014 to $76 \%$ in 2015 . Figure.1 shows an example of multifunctional terminal for automobile, which combines a GSM antenna coupling and a Near Field Communication system (NFC).

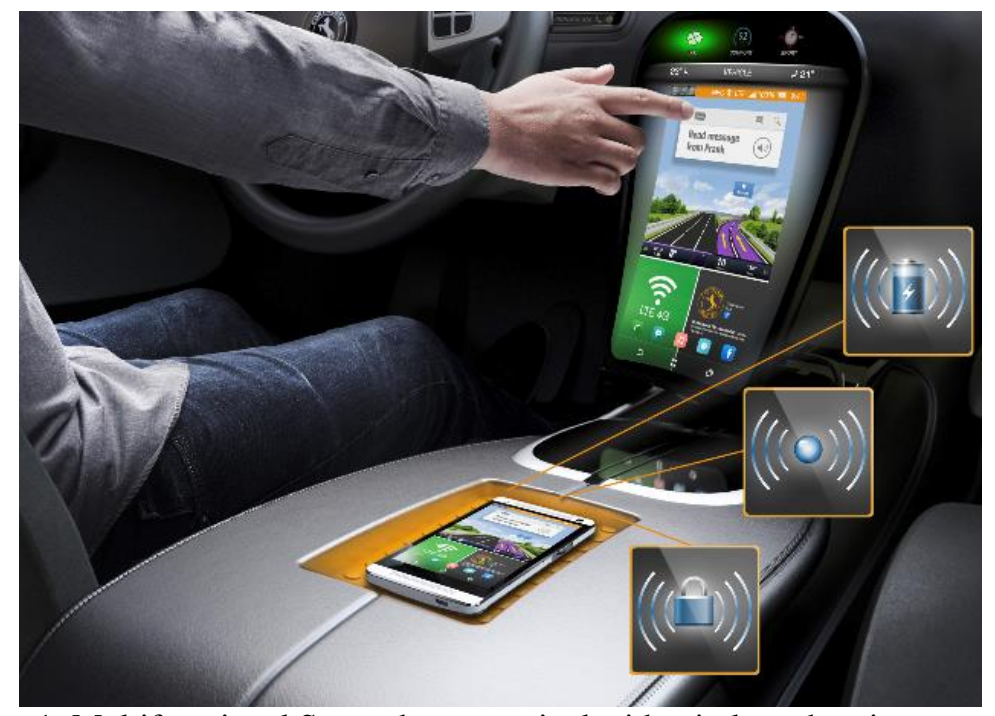

Fig. 1: Multifunctional Smartphone terminal with wireless charging system. 
On the market, alongside of the first standard "Qi" [1] working over low frequency range of $100 \mathrm{kHz}-200 \mathrm{kHz}$, the "Rezonance" standard (pronounced reh-zense) delivers up to $5 \mathrm{~W}$ at $6.78 \mathrm{MHz}$.

Compared to traditional charging with cable, wireless charging offers many benefits by:

- $\quad$ eliminating the need for power cords and reducing e-waste.

- eliminating the sparking hazard associated with conductive interconnections

- $\quad$ providing the requested power by charging intelligently the devices

- eliminating the risk of electrical shock when compared with conductive charging

However, it is important to analyze the biological compatibility of the wireless charging systems and to identify the conditions which may demonstrate compliance with safety guiltiness. The lack of knowledge and complete analysis about the absorption mechanism of wireless charging systems motivated this study.

The Interaction of wireless charging and human body can be impacted mainly by five parameters:

- distance to human body and coil orientation: absorption risks dercease when the separation distance increases between the transmitting coil and the human tissue.

- human body: penetration and specific absorption rate in human body depend on electrical proprieties (conductivity and permittivity) and thermal parameters of human tissues.

- $\quad$ charging power: exposure is strongly dependent on transmitting coil current.

- $\quad$ operating frequency: skin depth of a human tissue decreases as the operational frequency increases

- Smartphone structure: metallic and magnetic elements inside the mobile phones interact with the transmitting coil and could attenuate the incident fields.

\section{Applicable limitation levels and Specific Absorption Rate}

Many standards have been issued by different organizations like the International Commission on Non-Ionizing Radiation Protection (ICNIRP) [2] and Institute of Electrical and Electronic Engineers (IEEE) [3].

In order to protect against excessive tissue absorption, two limits have been set by the standards following a thorough review of all published scientific documents are: basic restrictions and reference levels. For Wireless Power Systems (WPS), the electromagnetic exposure is highly localized and the use of reference levels is not appropriate and leads to a high overestimation. Appropriately, in such situations respect of the localized basic restrictions should be applied. From $100 \mathrm{kHz}$ to $10 \mathrm{MHz}$, the physical quantities used to specify the basic restrictions are current density (J) and Specific Absorption Rate (SAR). Safety factors shall be applied to consider modelling uncertainties and biological variations. Moreover, precautionary measures should be taken to reduce the exposure if the exposure limits are exceeded.

According to the Poynting theorem [4], the power dissipated in the biological tissue can be determined with:

$$
\begin{aligned}
\operatorname{SAR}\left(I_{\text {coil }}, d, \theta, y\right) & =\int_{V} \omega\left(\left(1-\gamma_{E(y)}\right) \varepsilon^{\prime}\left|\vec{E}\left(d, \theta, I_{\text {coil }}\right)\right|^{2}+\left(1-\gamma_{H(y)}\right) \mu^{\prime}\left|\vec{H}\left(d, \theta, I_{\text {coil }}\right)\right|^{2}\right) d V \\
& +\int_{V} \sigma\left(1-\gamma_{E(y))}\left|\vec{E}\left(d, \theta, I_{\text {coil }}\right)\right|^{2} d V\right.
\end{aligned}
$$

The first item represents the power dissipated in the biological tissue due to electric $\left(\varepsilon^{\prime}\right)$ and magnetic losses $\mu^{\prime}$ and the second part is the power dissipated in a non-ideal conductance $(\sigma)$. The $\gamma_{H}$ and $\gamma_{E}$ are the attenuation factors due to the structure of the Smartphone on magnetic $(|\vec{H}|)$ and electric component $(|\vec{E}|)$. However, the magnetic field $|\vec{H}|$ produces a neglected power absorption in human body compared to the electric field $|\vec{E}|[5]$. The aim impacting mechanisms of the power absorption are shown in figure. 2.

From (1), the SAR is an estimation of the internal localized electric field in a defined volume of V (a cube of $10 \mathrm{~g}$ of human tissue as defined in ICNIRP standard). The SAR is can be estimated in the following manner [6] [7]:

$$
\operatorname{SAR}\left(I_{\text {coil }}, d, \theta, x\right)=\frac{\gamma_{E(x)} \sigma_{e f f}}{\rho} \int_{1 / 10 g}\left|\vec{E}\left(I_{\text {coil }}, d, \theta\right)\right|^{2} d V
$$


Where:

$\sigma_{e f f}$ : conductivity of human body tissue $(\mathrm{S} / \mathrm{m})$

$\rho$ : mass density of human body tissue $\left(\mathrm{kg} / \mathrm{m}^{3}\right)$

$\mathcal{E} "$ : permittivity of the human tissue $(\mathrm{F} / \mathrm{m})$

$\gamma_{E}(y)$ are the attenuation factor on electric field at position y

$\omega=2 \pi f$

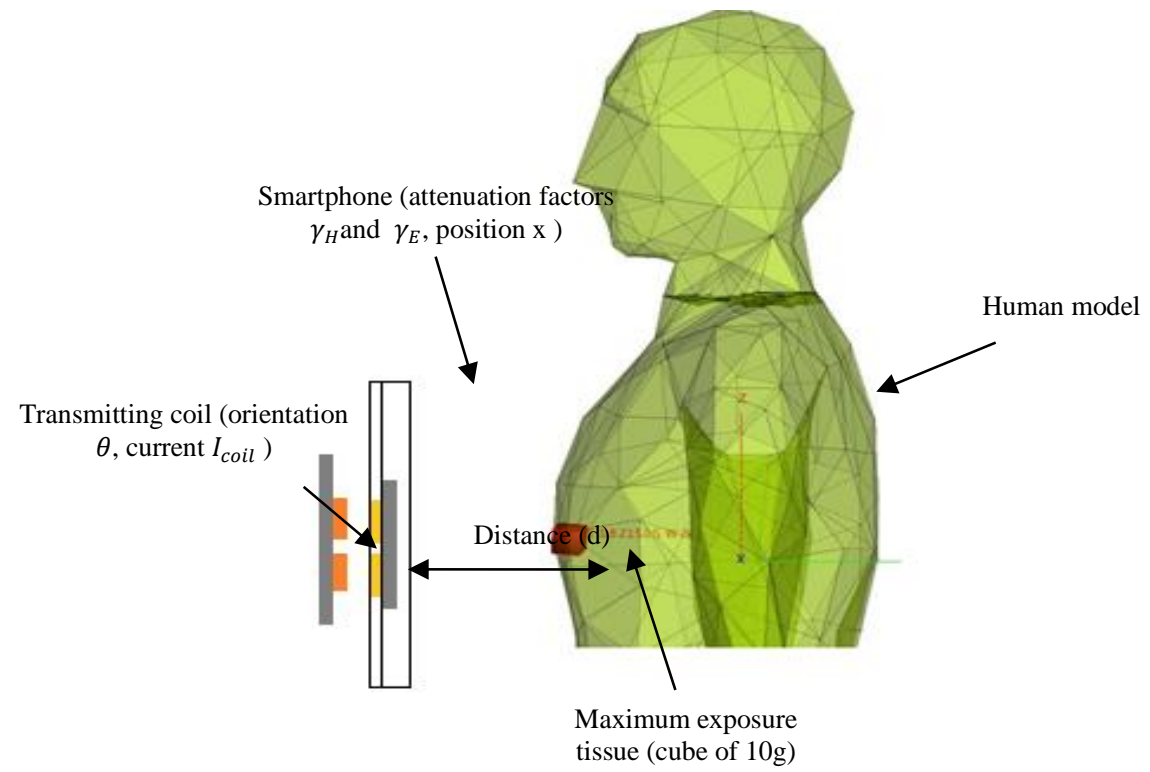

Fig. 2: main impacting parameters of EM human exposure.

The internal electric field and current density $\left(\mathrm{A} / \mathrm{m}^{2}\right)$ are related by Ohm's Law:

$$
\mathrm{J}=\sigma \mathrm{E}
$$

For the general public, the safety lowest limits are $0.08 \mathrm{~W} / \mathrm{kg}$ (SARavg) for whole body averaged and $2 \mathrm{~W} / \mathrm{Kg}$ for localized SAR10g. Localized SARA10g shall be average in any $10 \mathrm{~g}$ of contiguous tissue and for each point a cube with a defined mass is found.

In wireless charging systems, the electromagnetic field is emitted in two different conditions: pinging and charging mode. The pinging mode sends multiple signal pulses to detect and to adjust the highest charging efficiency of mobile device. Only when device has been detected and validated will power transfer starts. According to ICNIRP and IEEE, all SAR values are to be averaged over any six-minute period. Thus, the charging mode is considered as worst case emission.

The human body is composed of more than 45 different tissues having specific proprieties.

Table. 1: conductivity and permittivity of relevant human tissues at $100 \mathrm{kHz}$.

\begin{tabular}{|l|l|l|}
\hline & \multicolumn{1}{|c|}{ Conductivity $[\mathbf{S} / \mathbf{m}]$} & Permittivity $[\mathbf{F} / \mathbf{m}]$ \\
\hline muscle & 0.36 & 7800 \\
\hline blood & 0.70348 & 5102.7 \\
\hline Fat & 0.025066 & 66.295 \\
\hline Nerve & 0.082344 & 4726 \\
\hline
\end{tabular}

The interaction of EM field with human tissues is highly depending on frequency. The table.1 shows the conductivity and relative permittivity for relevant human tissues: muscle, blood, fat and nerve. 


\section{Experimental validation of the wireless power source}

In order to validate electromagnetically the numerical model against laboratory measurements, H-field levels (magnetic) were measured and compared to the simulations results. In the numerical simulation, the generated magnetic fields are calculated by full-wave analysis solver based on hybrid method of Moments and Finite Element Method (FEKO). The $100 \mathrm{kHz}$ coils have a rectangular shape and consist of one layer. The wires were modeled as prefect conductive with a diameter of $0.6 \mathrm{~mm}$ and the number of turns is 10 . The inner and outer diameters of the coils are Din $=19.5 \mathrm{~mm}$ and Dout= $45.2 \mathrm{~mm}$, respectively. The design contains 3 different coils, placed alongside each other with a displacement of with $49.2 \mathrm{~mm}$ between their centers.
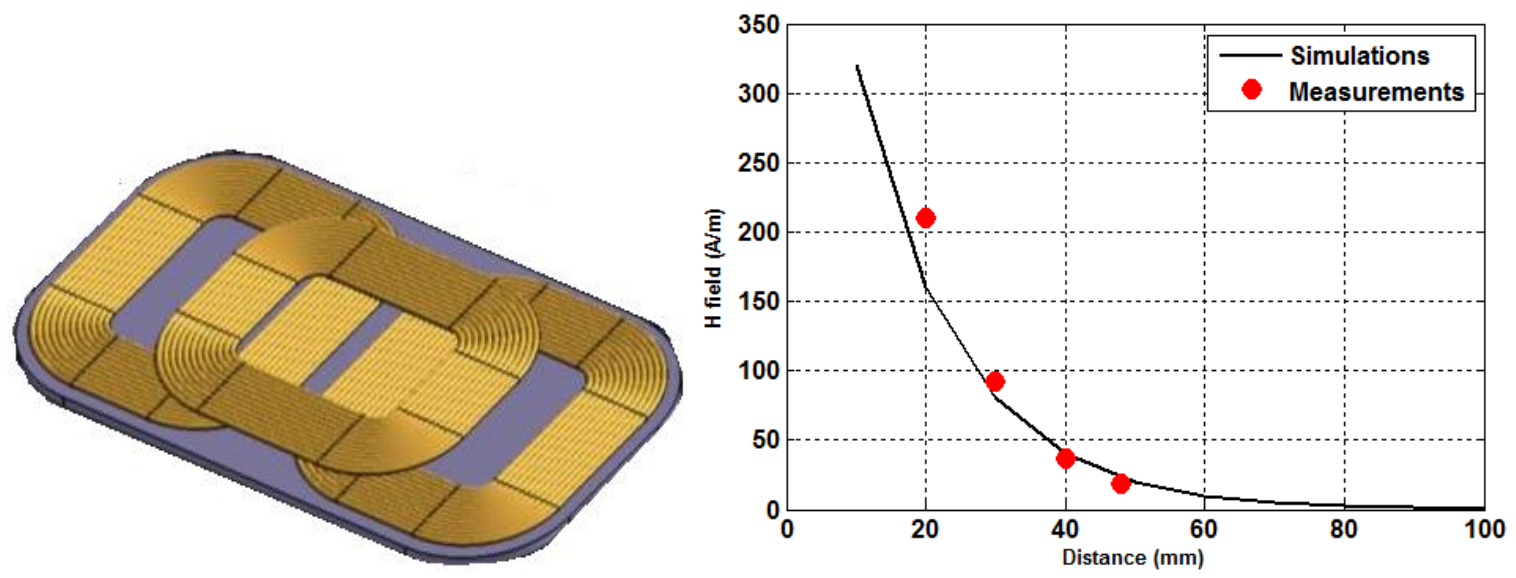

Fig. 3: from left to right: numerical model of the wireless charger coils and magnetic field along the distance for a coil current of 1 Arms at $100 \mathrm{kHz}$

The calculated and measured field distributions are shown in Fig.3. The field level of calculated results agrees well with measurements and shows a difference within 5\%. Thus, the validly of the field model has been confirmed.

The metallic and magnetic elements inside the mobile phones interact with the transmitting coils. The electromagnetic fields attenuation is then analyzed at different phone positions (from $\mathrm{y}=-20 \mathrm{~mm}$ to $\mathrm{y}=+20 \mathrm{~mm}$ with a step of $10 \mathrm{~mm}$ ), in order to estimate the attenuation factors $\gamma_{E}(y)$ and $\gamma_{H}(y)$. Comparisons are performed over 10 different Smartphone's, their E and $\mathrm{H}$ fields are analyzed and only the maximum and minimum attenuation factors are reported in Fig.4.

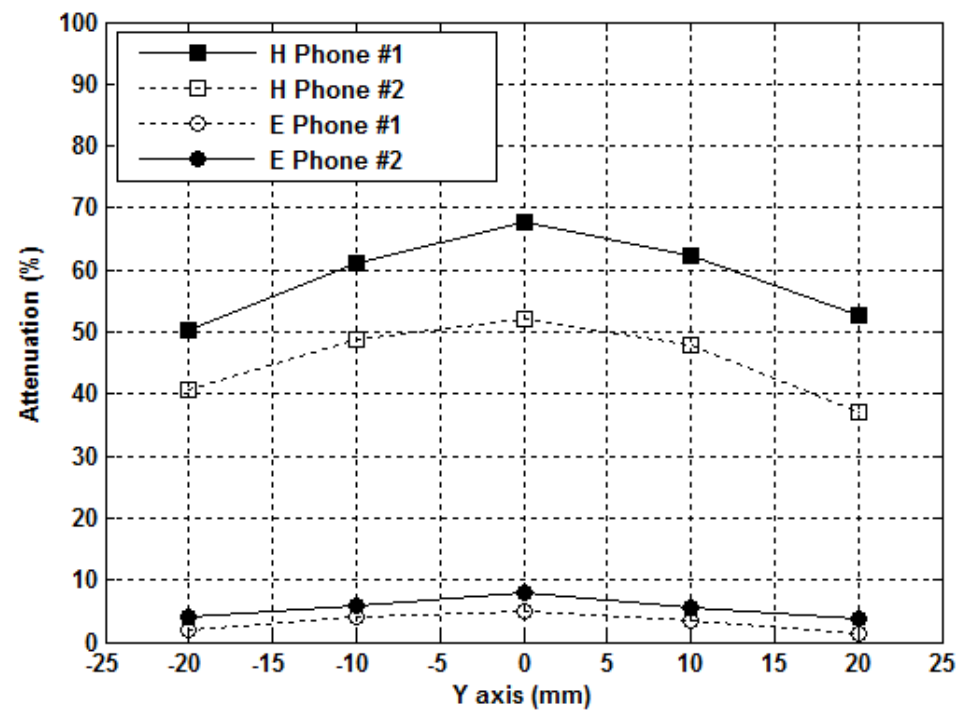

Fig. 4: measurement of the phone attenuations. 
The presence of the phone on the transmitting coils decreases the H-field by about $50 \%$ to $70 \%$. The mobile's comings from different manufacturers do not feature the same attenuation levels. The difference on the $\mathrm{H}$-field is $20 \%$ higher for the phone\#1 compared to phone\#2. However, it is important to emphasize that the E-field levels do not change significantly (less than $8 \%$ at $0 \mathrm{~mm}$ ). Therefore the maximum human exposure can be obtained with $\gamma_{E}(y+/-20 \mathrm{~mm})=4 \%$ and $\gamma_{H}(y+/-20 m m)=38 \%$.

\section{SAR assessment}

The SAR is a very important parameter related to human body interaction. High-resolution computations are used for an anatomically representative model of the human body and shown in fig.5. The hybrid used method is particularly suitable for complex inhomogeneous structures like the interaction between electromagnetic field and human body.

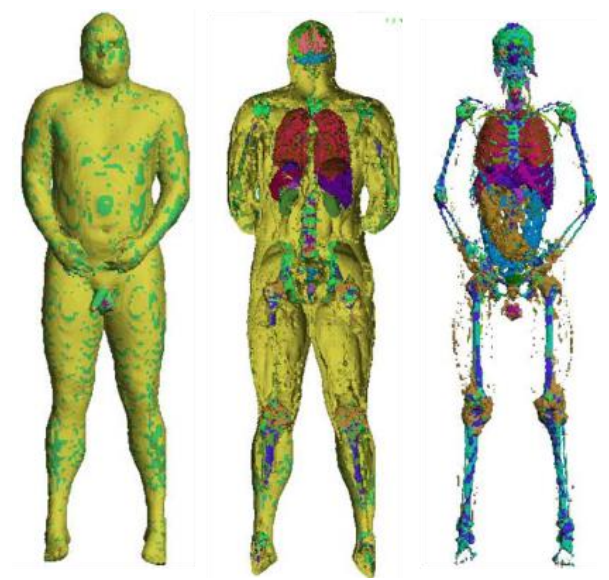

Fig. 5: volume rendered images of a male human body (from left to right): outside surface, the internal skeleton and the skeleton and internal organs.

The numerical human model is composed mainly of muscle-equivalent tissue and divided into more than 4000 cells. The transmitting coil is located in front of the human body and placed at two different distances from the human model: $10 \mathrm{~mm}$ and $20 \mathrm{~mm}$. The $\mathrm{E}$ and $\mathrm{H}$ fields have been evaluated using full anatomical model at $100 \mathrm{kHz}$ and compared to $10 \mathrm{MHz}$ with the validated model of the wireless power transfer. Fig.6 show the $\mathrm{H}$ and E-field distribution at $10 \mathrm{~mm}$ from the full models at $100 \mathrm{KHz}$ and $10 \mathrm{MHz}$ when the current of the transmitting antenna is $2.5 \mathrm{Arms}$. For both frequencies, it can be seen clearly that the magnetic field is not impacted by the presence of the human body. However, the asymmetries of human tissues impact strongly the electric field at $10 \mathrm{MHz}$. For equivalent magnetic field intensity, the generated electric field from $10 \mathrm{MHz}$ is 10 to 15 times higher than from $100 \mathrm{kHz}$.
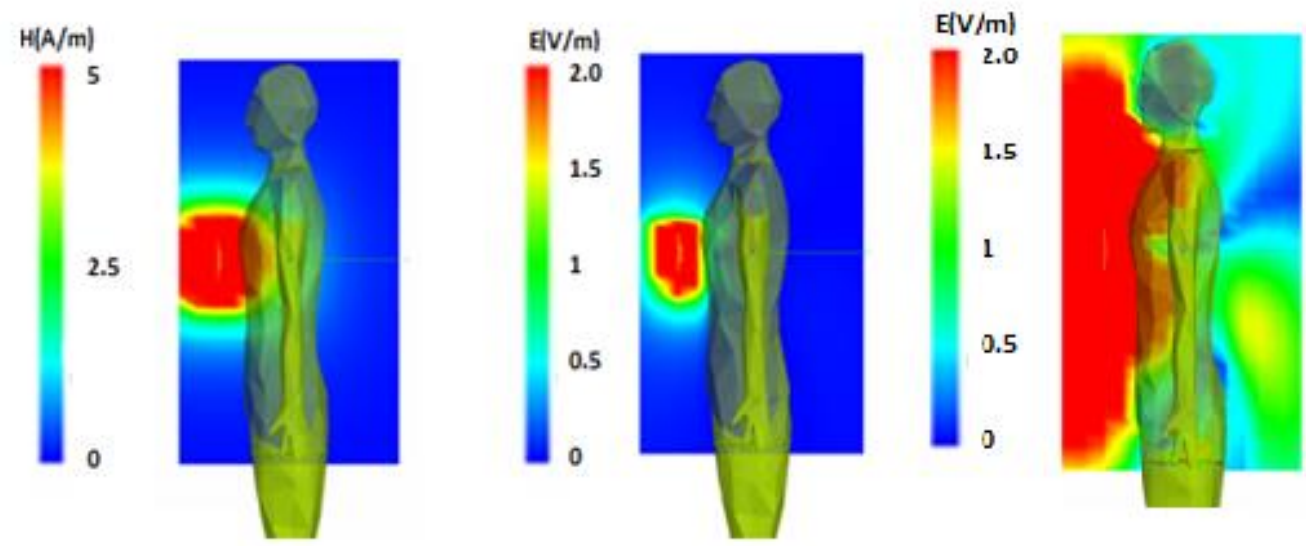

Fig. 6: field distribution of the wireless power transfer system with the human model (from left to right): Magnetic, electric field at $100 \mathrm{kHz}$ and electric field at $10 \mathrm{MHz}$. 
Comparing different coil orientations, the highest SAR levels are most restrictive at coronal plane $\left(\theta=0^{\circ}\right.$ placed horizontally to human body) because the transmitting coil exposes biggest area of human tissue in this orientation. The estimated SAR at coronal is 34 times higher than axial orientation $\left(\theta=90^{\circ}\right.$ placed vertically to human body).

To provide context for the coil current $I_{\text {coil }}$ at which the SAR thresholds were achieved, the SAR was estimated at different $I_{\text {coil }}$ from 1 Arms to 5 Arms.

The summazried SAR10g results are shown in Fig.7. Increasing the distance between the source and the human model from $10 \mathrm{~mm}$ to $20 \mathrm{~mm}$ causes the SAR decrease by almost $81 \%$ at the frequencies of intrest. At $100 \mathrm{kHz}$, the maximum peak SAR $10 \mathrm{~g}$ of $0,4 \mathrm{~W} / \mathrm{Kg}$ was obtained for a transmitting current of 10 Arms at the closest distance to the human body (10mm).
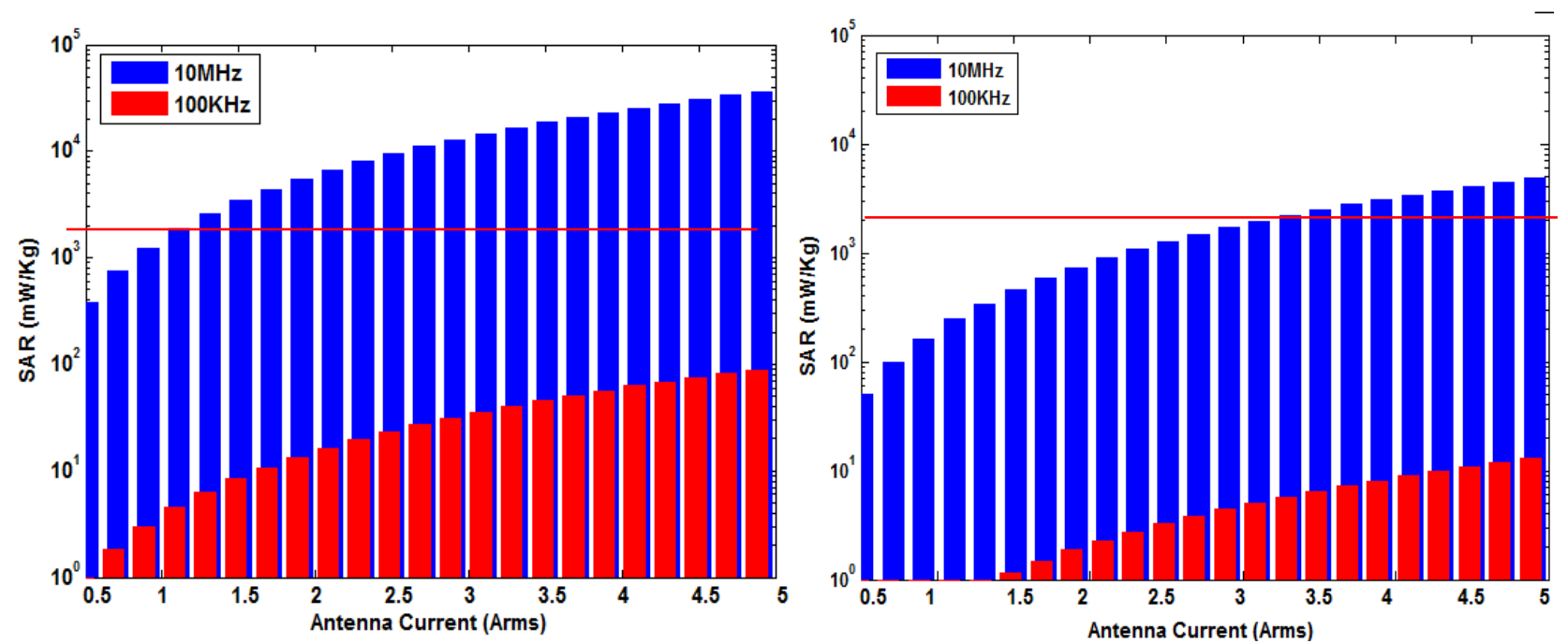

Fig. 7: the summazried SAR10g results (from left to right): at $10 \mathrm{~mm}$ and $20 \mathrm{~mm}$ distance to human model.

Using a lumped circuit model with a coupling factor $\mathrm{k}=0.2$, the maximum charging power at which SAR reaches $0.4 \mathrm{~W} / \mathrm{Kg}$ is $65 \mathrm{~W}$ at $100 \mathrm{kHz}$. These results show that the inductive link at $100 \mathrm{kHz}$ leads a small energy absorption and tissue heating in human body and confirm the compliance of wireless charging with RF exposure limits can be achieved at extremely high power charging.

However, a deep evaluation at various levels needs to be given careful consideration to assess the exposure impacts at $10 \mathrm{MHz}$. As shown in fig.8, the chance of absorption of radiation increases at high power charging, this can cause adverse health problems. Thus, it is mandatory to attenuate the incident electric field by placing the charging coil at least at $20 \mathrm{~mm}$ distance from the human tissues and limiting the coil current at 2Arms.

A direct calculation of the expected temperature rise in human tissue exposed to E-fields can be made from the equation (4):

$$
\mathrm{aT}=\mathrm{SAR} 10 \mathrm{~g} \mathrm{t} / \mathrm{C}
$$

where:

$\mathrm{C}$ : specific heat capacity $\left(\mathrm{J} / \mathrm{Kg}{ }^{\circ} \mathrm{C}\right)$

$\mathrm{T}$ : exposure time in seconds.

The temperature computation provides information on the maximum temperature rise during the wireless charging and within the human body exposed to the EM fields [8] [9]. Fig.8 shows the temperature-rise distribution at for the muscle at $10 \mathrm{~mm}$ distance. The temperature rise was estimated with a specific capacity value of $3686 \mathrm{~J} / \mathrm{Kg}{ }^{\circ} \mathrm{C}$. A charging power of 5 Watts (typical charging power for Smartphone's) has been used to evaluate the temperature rise, which corresponds to a coil current of 2 Arms at $100 \mathrm{kHz}$ and $1.2 \mathrm{Arms}$ at $10 \mathrm{MHz}$. 


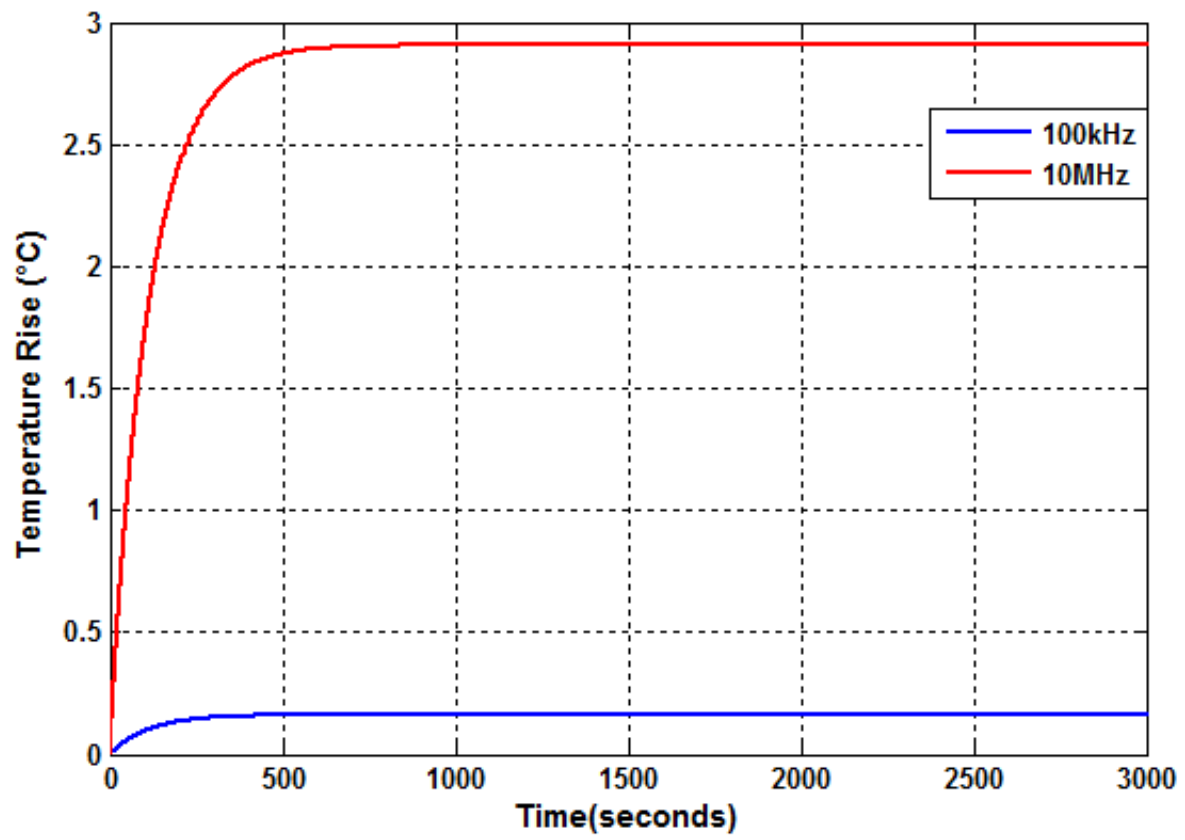

Fig. 8: Calculation of temperature change during charging at $100 \mathrm{kHz}$ and $10 \mathrm{MHz}$.

The fig. 8 shows that the temperature rise increases exponentially over the 500 seconds, then the rate of temperature becomes constant in function of time. The maximum temperature elevations obtained at $100 \mathrm{kHz}$ and $10 \mathrm{MHz}$ are $0.14^{\circ} \mathrm{C}$ and $2.9^{\circ} \mathrm{C}$, respectively with the same received power (5Watts).

\section{Conclusion}

The exposure of a person in the reactive charging field has been analyzed and investigated in this study with an objective to provide recommendations and a full risk assessment with respect of human safety limits.

Measurements and calculations were done for generated electromagnetic field and specific absorption rate (SAR) in human tissues. The exposure is strongly dependent on distance, reactive power, coil orientation and frequency. Charging systems at $10 \mathrm{MHz}$ possesses the highest SAR values and exceeds the safety limit of $2 \mathrm{~W} / \mathrm{Kg}$ at $10 \mathrm{~mm}$. Therefore, health risk exists at high frequency systems and special care should be taken into account. On this basis, a set of recommendations shall be proposed by the standard for reduction of the SAR on human tissues.

\section{References}

[1] Wireless Power Consortium, System Description Wireless Power Transfer, Volume I: Low Power, Part 1: Interface Definition. version 1.1.1, 2012.

[2] International Commission on Non-Ionizing Radiation Protection (ICNIRP), "Guidelines for limiting exposure to timevarying electric, magnetic and electromagnetic fields (up to $300 \mathrm{GHz}$ )," Health Phys., vol. 74, pp. 492-522, 1998.

[3] "IEEE Standard for safety Levels with Respect to Human Exposure to Radio Frequency Electromagnetic fields, $3 \mathrm{kHz}$ to $300 \mathrm{GHz}$," C95.1-2005.

[4] C. Picher, J. Anguera, A. Andujar, and C. Puente, "Analysis of the human head interaction in handset Antennas with Slotted ground Planes," IEEE Antennas and Propagation Magazine, vol. 54, pp. 39-56, 2012.

[5] N. Kuster and Q. Balzano, "Energy Absoption Mechanism by Biological Bodies in the Near Field of Dipole Antenna Above 300MHz," IEEE Transaction On vehicular Technlogu, vol. 41, pp. 17-23, 1992.

[6] I. Laakso, S. Tsuchida, A. Hirata, and Y. Kamimura, "Evaluation of SAR in a human body model due to wireless power transmission in the $10 \mathrm{MHz}$ band," Phys. Med. Biol., vol. 57, pp. 4991-5002, 2012.

[7] T. Sunohora, I. Laasko, and T. Onishi "Induced field and SAR in human body model due to wireless power transfer system with induction coupling," in EMC'14, Tokyo, pp. 449-452. 
[8] A. Fazlul, "A study of Specific Absorption Rate (SAR) Due to Non-Ionizing Radiation from wireless/Telecommunication in Bangladesh," American Journal of Physics and Applications, pp. 104-110, 2013.

[9] P. R. Wainwright, "The relationship of temperature rise to specific absorption rate and current in the human leg for exposure to electromagnetic radiation in the high frequency band," INSTITUTE OF PHYSICS PUBLISHING, 2003.

[10] P. Bernardi, M. Cavagnaro, S. Pisa, and, E. Piuzz, "Specific Absorption Rate and Temperature Elevation in a Subject Exposed in the Far-Field of Radio-Frequency Sources Operating in the 10-900-MHz Range," IEEE TRANSACTIONS ON BIOMEDICAL ENGINEERING, 2003.

[11] W. Kang, Z. Alexander, and Y. Park "Exposure assessment for wireless multi-phone charger," in EMC'14, Tokyo, pp. 198-201.

[12] E. Waffenschmidt, "Wireless power for mobile devices," in Proc. IEEE 33 rd Int. Telecommun. Energy Conf., pp. 1-9, 2011.

[13] P. J. Riu and K. R. Foster, "Heating of tissue by near-field exposure to a dipole: a model analysis," IEEE Trans. Biomedi. Eng., vol. 46, no. 8, pp. 911-917, 1999.

[14] D. Schneider, “A critical look at wireless power,” IEEE Spectr., vol. 47, no. 5, pp. 34-39, 2010. 